\title{
Endothelial-derived FGF2 contributes to the progression of pulmonary hypertension in humans and rodents
}

\author{
Mohamed Izikki, ${ }^{1}$ Christophe Guignabert, ${ }^{1}$ Elie Fadel,, ${ }^{2}$ Marc Humbert, ${ }^{3}$ \\ Ly Tu, ${ }^{1}$ Patricia Zadigue, ${ }^{1}$ Philippe Dartevelle, ${ }^{2}$ Gerald Simonneau, ${ }^{3}$ \\ Serge Adnot, ${ }^{1}$ Bernard Maitre, ${ }^{1}$ Bernadette Raffestin, ${ }^{1}$ and Saadia Eddahibi'
}

\begin{abstract}
${ }^{1}$ INSERM U841, Institut Mondor de Recherche Biomédicale, Faculté de Médecine, Créteil, France. ${ }^{2}$ UPRES EA2705, Service de Chirurgie Thoracique, Vasculaire et de Transplantation Cardiopulmonaire, Hôpital Marie Lannelongue, Le Plessis Robinson, France. ${ }^{3}$ INSERM U764, Service de Pneumologie, Centre National de Référence de l'Hypertension Artérielle Pulmonaire, Hôpital Antoine-Béclère, Assistance-Publique Hôpitaux de Paris, Clamart, France.
\end{abstract}

Pulmonary hypertension (PH) is a progressive, lethal lung disease characterized by pulmonary artery SMC (PA-SMC) hyperplasia leading to right-sided heart failure. Molecular events originating in pulmonary ECs (P-ECs) may contribute to the PA-SMC hyperplasia in PH. Thus, we exposed cultured human PA-SMC to medium conditioned by P-EC from patients with idiopathic PH (IPH) or controls and found that IPH P-ECconditioned medium increased PA-SMC proliferation more than control P-EC medium. Levels of FGF2 were increased in the medium of IPH P-ECs over controls, while there was no detectable difference in TGF- $\beta 1$, PDGF-BB, or EGF levels. No difference in FGF2-induced proliferation or FGF receptor type 1 (FGFR1) mRNA levels was detected between IPH and control PA-SMCs. Knockdown of FGF2 in P-EC using siRNA reduced the PA-SMC growth-stimulating effects of IPH P-EC medium by $60 \%$ and control P-EC medium by $10 \%$. In situ hybridization showed FGF2 overproduction predominantly in the remodeled vascular endothelium of lungs from patients with IPH. Repeated intravenous FGF2-siRNA administration abolished lung FGF2 production, both preventing and nearly reversing a rat model of PH. Similarly, pharmacological FGFR1 inhibition with SU5402 reversed established PH in the same model. Thus, endothelial FGF2 is overproduced in IPH and contributes to SMC hyperplasia in IPH, identifying FGF2 as a promising target for new treatments against PH.

\section{Introduction}

Pulmonary hypertension (PH) develops either as a complication of various disease states or as an idiopathic condition (1). Mutations in the bone morphogenic protein receptor type 2 (BMPR2) have been linked to familial cases of idiopathic PH (IPH), but the molecular basis for common sporadic or associated forms is unknown. A major component of the pulmonary vessel remodeling process that leads to IPH is proliferation of pulmonary artery SMCs (PA-SMCs) (2), which may result either from inherent characteristics of PA-SMCs or from dysregulation of molecular events governing PA-SMC growth, such as signals originating in pulmonary ECs (P-ECs) (3).

One critical aspect of vessel development and maturation is the release by ECs of growth factors that recruit mural cells and stabilize the vessel wall (4). Thus, endothelium-derived growth factors that modulate SMC migration and proliferation during vessel maturation may be involved in abnormal vessel remodeling. Recent evidence indicates a role for dysregulation of these molecular pathways governing vessel maturation in the pulmonary vessel remodeling process that characterizes $\mathrm{PH}(1,2)$. In previous studies, we found that culture medium from P-ECs induced proliferation of PA-SMCs and that this effect was considerably stronger when we used P-ECs from patients with IPH rather than from controls

Conflict of interest: The authors have declared that no conflict of interest exists. Nonstandard abbreviations used: ET-1, endothelin-1; FGFR1, FGF receptor type 1; FM, fully muscular; FM+, obliterated; IPH, idiopathic pulmonary hypertension; MCT, monocrotaline; NM, nonmuscular; $\mathrm{PAP}$, pulmonary artery pressure; PA-SMC, pulmonary artery SMC; PCNA, proliferating cell nuclear antigen; P-EC, pulmonary EC; $\mathrm{PH}$, pulmonary hypertension; $\mathrm{PM}$, partially muscular; S, interventricular septum. Citation for this article: J. Clin. Invest. 119:512-523 (2009). doi:10.1172/JCI35070.
$(3,5)$. The mediators involved in this process were not identified, although part of the proliferative effect seemed related to overproduction of serotonin and endothelin-1 (ET-1) $(3,5)$. Moreover, we previously reported that the release of endothelium-derived growth factors capable of recruiting vessel-wall cells was influenced by the angiopoietin/Tie2 pathway (5). In the present study, we investigated whether growth factors known to participate in vessel development and EC/pericyte interactions were involved in this abnormal crosstalk between P-ECs and PA-SMCs during the progression of $\mathrm{PH}$. We focused on FGF2 because, among the main growth factors expressed by ECs (including PDGF, TGF- $\beta$, EGF, and FGF2), only FGF2 was released in excessive amounts by P-ECs of patients with IPH. FGF2, a member of a large family of heparinbinding growth factors $(6,7)$, is synthesized by several cell types including tumor cells, fibroblasts, ECs, and macrophages (8-10). Moreover, FGF2 can be sequestered and stored as a complex in the extracellular matrix, then released by proteolytic processes to bind and activate cell targets, thereby promoting mitogenesis (11-14). FGF2 exerts its biological activity by binding to high-affinity tyrosine kinase FGF receptors (FGFRs), such as FGFR1, expressed on the surface of vascular cells (15-18).

Increased lung and circulating FGF2 levels have been reported in both experimental and human PH. Abnormally high levels of FGF2 were found in the blood of $51 \%$ and in the urine of $21 \%$ of patients with IPH (19) and in 2 animal models, a lamb model of PH developed by inserting an aortopulmonary vascular bypass graft (20) and the rat model of monocrotaline-induced (MCTinduced) PH (8). However, the importance of the FGF2 increase in initiating and perpetuating the disease remains unclear. 
A
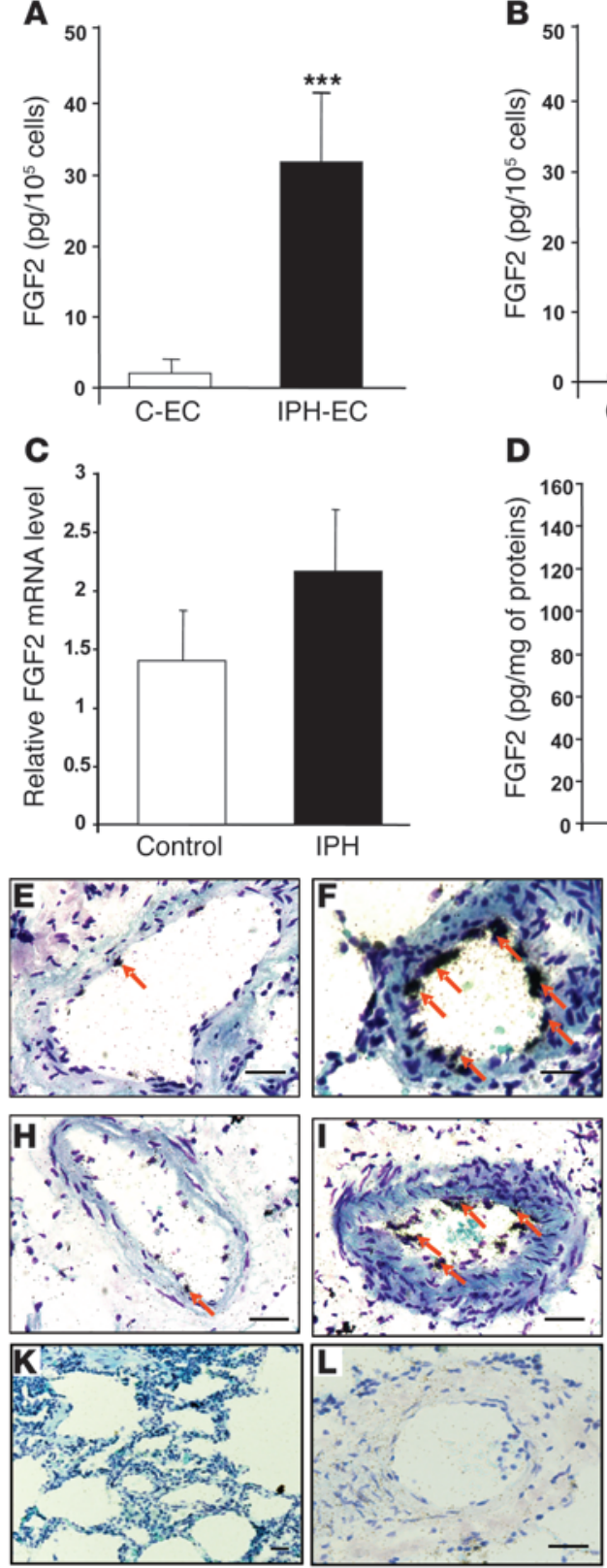

B

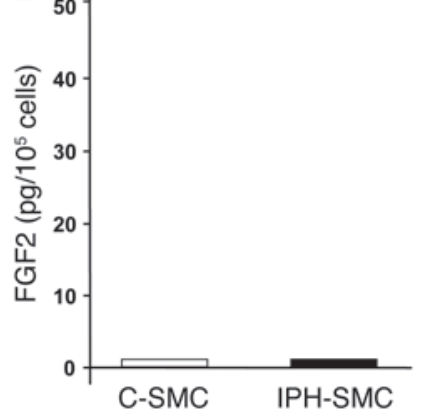

D
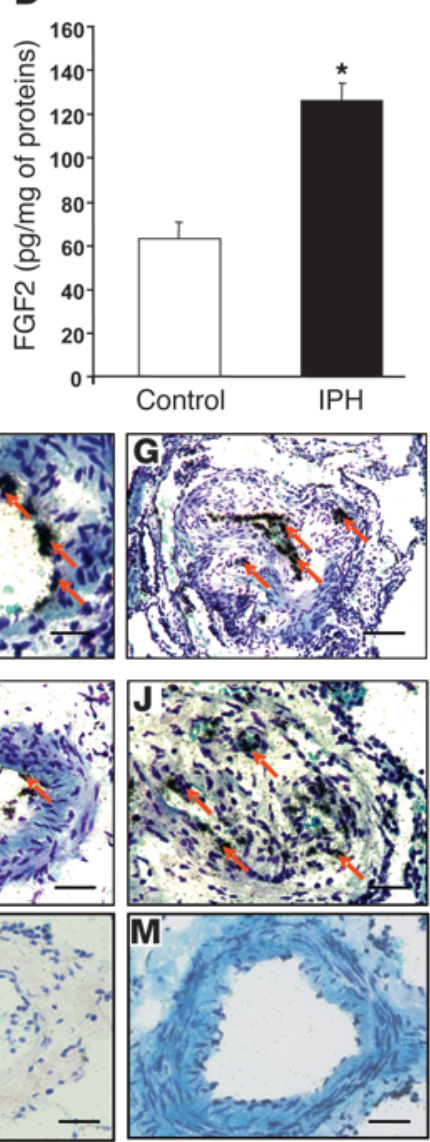

Figure 1

FGF2 expression in cultured P-ECs, PA-SMCs, and lungs from patients with IPH. (A) FGF2 expression by P-ECs and (B) PA-SMCs from controls (C-EC and C-SMC, respectively) and from patients with IPH (IPH-EC and IPH-SMC, respectively). Values are mean \pm SEM from 5 controls and 5 patients with IPH. (C) FGF2 mRNA levels and (D) FGF2 protein levels in lung tissues from controls and patients with IPH. Values are mean \pm SEM from 12 controls and 14 patients for mRNA levels and 5 controls and 5 patients for protein levels. (E-M) In situ hybridization in human pulmonary arteries from controls $(\mathbf{E}$ and $\mathbf{H})$ and from patients with IPH $(\mathbf{F}, \mathbf{G}, \mathbf{I}, \mathbf{J})$ using $3^{\prime}-$ $\left[{ }^{35} \mathrm{~S}\right]$-labeled oligonucleotide probes specific for human FGF2 or using a sense probe (K-M). Arrows indicate the localization of FGF2 mRNA in the vascular endothelium of muscular pulmonary arteries. ${ }^{\star} P<0.05$; ${ }^{\star \star \star} P<0.001$ vs. P-ECs, PA-SMCs, or lungs from control subjects. Scale bars: $100 \mu \mathrm{m}$.

from controls (Figure 1A). In contrast, no differences were found between the 2 groups regarding the levels of TGF- $\beta$, PDGF, or EGF (Table 1). Measurements of FGF2 protein in cell culture medium showed greater FGF2 protein production by P-ECs than by PA-SMCs (Figure 1A). Moreover, adding exogenous human leukocyte elastase to cultured P-ECs resulted in significantly greater FGF2 release from the extracellular matrix of IPH P-ECs than that of control P-ECs (data not shown).

FGF2 expression in lungs from patients with IPH and controls. As shown in Figure 1B, whereas mRNA expression showed only a small nonsignificant increase, a marked increase in FGF2 protein was found in lung homogenates from patients with IPH compared with controls. In situ hybridization on lung sections showed that FGF2 mRNA was predominantly located in P-ECs of pulmonary arteries in both the patients with IPH (Figure 1, F-J) and the controls (Figure 1, E and H). In agreement with the PCR and ELISA results, in situ hybridization showed that P-ECs from patients with IPH generated a strong FGF2 signal, which predominated in remodeled pulmonary arteries. The FGF2 signal was weak in vessels from controls. RNA in situ hybridization with a sense probe produced little or no signal compared with that using an antisense probe (Figure 1, K-M).

FGF2 expression by $P$-ECs and effect on proliferation of PA-SMCs. To evaluate the contribution of endothelium-derived FGF2 to the stimulation of PA-SMC growth, we treated cultured

The objective of this study was to investigate the role for FGF2 in crosstalk between P-ECs and PA-SMCs in humans with IPH as well as in the development and perpetuation of experimental PH in rats. To this end, we developed siRNA to induce knockdown of human FGF2 in experiments designed to assess interactions between P-ECs and PA-SMCs from patients with IPH and from controls. We then conducted experiments in rats to determine whether repeated administration of siRNA directed against rat FGF2 and inhibition of FGFRs prevented and/or reversed MCTinduced pulmonary vascular remodeling and $\mathrm{PH}$.

\section{Results}

Growth factors released by ECs from patients with IPH and controls. Levels of FGF2 protein were markedly increased in the medium of quiescent P-ECs from patients with IPH compared with those
P-ECs from lungs of patients with IPH or controls with siRNA against human FGF2 (FGF2-siRNA) or with a scrambled sequence of this siRNA (control siRNA). We then added the treated P-EC medium to cultured PA-SMCs. PA-SMC growth responses were measured using $\left[{ }^{3} \mathrm{H}\right]$ thymidine incorporation. We first determined that P-EC transfection by FGF2-siRNA was effective in inhibiting FGF2 expression compared with transfection by the scrambled siRNA sequence. FGF2 mRNA levels were reduced by $10 \%$ in P-ECs from controls and by 55\% in P-ECs from patients with IPH (Figure 2). Adding P-EC medium stimulated PA-SMC growth; however, the growth increase was larger when medium from IPH P-ECs was used compared with medium from control P-ECs (Figure 3A). PA-SMC growth responses were similar with medium of untreated P-ECs and medium of P-ECs treated with control siRNA (Figure 3, A and B). In contrast, as shown in Figure 3, B and C, PA-SMC growth was 


\section{Table 1}

Growth factors released by P-ECs from controls and from patients with IPH

$\begin{array}{lcc} & \text { Controls }(\boldsymbol{n}=\mathbf{5}) & \text { IPH }(\boldsymbol{n}=\mathbf{5}) \\ \text { FGF2 }(\mathrm{pg} / \mathrm{ml}) & 2.10 \pm 1.05 & 30.2 \pm 7.90^{A} \\ \text { PDGF-BB }(\mathrm{pg} / \mathrm{ml}) & 2.40 \pm 0.50 & 2.60 \pm 0.27 \\ \text { EGF }(\mathrm{pg} / \mathrm{ml}) & 0.75 \pm 0.15 & 0.80 \pm 0.30 \\ \text { TGF- } \beta 1(\mathrm{pg} / \mathrm{ml}) & 163.3 \pm 10.9 & 191.6 \pm 8.5\end{array}$

Growth factor levels in conditioned medium from cultures of P-ECs from patients with IPH or controls after 24 hours incubation. Each bar represents mean \pm SEM. ${ }^{A} P<0.01$ vs. P-ECs from control subjects.

$60 \%$ lower in IPH P-ECs treated with FGF2-siRNA than in IPH P-ECs treated with control siRNA. This difference was only $10 \%$ when P-ECs from controls were used.

Exogenous FGF2 induced a mitogenic effect that was similar in PA-SMCs from patients with IPH and from controls, and this effect was not altered by EGFR or PDGFR inhibitors. In contrast, pretreatment with the FGFR1 antagonist SU5402 in a dose of $10^{-5} \mathrm{M}$ completely inhibited FGF2-induced proliferation (Figure 3D). Similarly, using real-time quantitative PCR, we found no significant difference in FGFR1 mRNA levels between IPH PA-SMCs and control PA-SMCs (2.75 \pm 0.5 vs. $2.68 \pm 0.4$, respectively, $n=5 ; P=\mathrm{NS}$ ).

FGF2 expression in MCT-injected rats after treatment with FGF2-siRNA, scrambled siRNA sequence, or vebicle. To evaluate the effect of FGF2siRNA in MCT-injected rats, FGF2 expression was measured in rat lungs at various times after MCT injection. FGF2 mRNA levels showed an early increase after 12 hours, which continued until day 5 , then further increases on day 15 and day 21 after MCT administration. FGF2 mRNA levels were higher in MCT-injected rats than in control rats (Figure 4).

In pretreatment, the first FGF2-siRNA injection compared with control siRNA blunted the early increase in FGF2 mRNA and protein observed 12 hours after MCT injection. A larger FGF2 reduction was seen 120 hours after MCT administration in the animals given 2 FGF2-siRNA injections versus 2 control siRNA injections (Figure 5A). Similarly, on day 21 after MCT administration, lung FGF2 mRNA and protein levels were markedly reduced in rats treated with 2 or 4 injections of FGF2-siRNA compared with rats treated with 2 or 4 injections of control siRNA. There were no significant differences for the decreases in FGF2 mRNA and protein between 2 and 4 FGF2-siRNA injections (Figure 5B).

For treatment, 2 injections of FGF2-siRNA given 21 days after the MCT injection induced a 2-fold decrease in lung levels of FGF2 mRNA and protein, compared with control siRNA (data not shown).

Evaluation of the efficacy of FGF2-siRNA pretreatment in MCT-induced $\mathrm{PH}$. In rats given control siRNA before the MCT injection, severe $\mathrm{PH}$ was noted on day 21, with marked increases in pulmonary artery pressure (PAP) (Figure 6A), RV hypertrophy assessed by the ratio of RV weight over $L V$ and interventricular septum (S) weight $(\mathrm{RV} /[\mathrm{LV}+\mathrm{S}])$ (Figure $6 \mathrm{~B})$, and distal pulmonary artery muscularization (Figure 6C) compared with control rats injected with saline instead of MCT. In contrast, pretreatment with FGF2-siRNA before the MCT injection markedly attenuated the severity of $\mathrm{PH}$ on day 21, as shown by marked decreases in PAP, RV/(LV + S), and distal artery muscularization. There were no significant differences between 2 and 4 FGF2-siRNA injections.
MCT-induced PH in rats is related to PA-SMC proliferation, collagen accumulation, and inflammation (21-24). Therefore, we evaluated the effect of FGF2 knockdown on these 3 events. Proliferating cell nuclear antigen (PCNA) immunohistochemistry results indicated that FGF2-siRNA markedly reduced SMC proliferation within the arterial wall (Figure 6, G-I). Moreover, FGF2siRNA reduced both the accumulation of collagen fibers stained blue by Masson trichrome stain (Figure 6, J-L) and the infiltration of macrophages as assessed by immunohistochemistry using the macrophage-specific marker CD68.

Evaluation of the efficacy of FGF2-siRNA treatment in MCT-induced PH. In rats treated with 2 FGF2-siRNA injections on days 21 and 25 after the MCT injection, evaluation on day 42 showed marked decreases in PAP (Figure 7A), RV/(LV + S) (Figure 7B), and distal artery muscularization (Figure $7 \mathrm{C}$ ) compared with rats given 2 injections of control siRNA. Systemic artery pressure and heart rate on day 42 were not significantly different from the values in control rats given saline instead of MCT (data not shown).

FGF2-siRNA injections decreased PA-SMC proliferation (Figure 7, F and G), collagen fiber accumulation (Figure 7, H and I), and macrophage infiltration (Figure 7, J and K) compared with injections of control siRNA.

Reversal of MCT-induced PH with the FGFR1 inhibitor SU5402. To confirm that the decreases in pulmonary vascular alterations and PH associated with FGF2-siRNA treatment were related to FGF2 knockdown, indicating a key role for FGF2 overproduction in $\mathrm{PH}$, we investigated to determine whether the selective FGFR1 inhibitor SU5402 prevented and/or reversed PH induced by MCT in rats. In rats treated with SU5402 on days 21 to 42 after the MCT injection, evaluations on day 42 showed marked decreases in PAP, $\mathrm{RV} /(\mathrm{LV}+\mathrm{S})$, and distal artery muscularization compared with rats treated with the vehicle (saline) (Figure 8).

\section{Discussion}

The present results show that dysregulated FGF2 signaling contributes to pulmonary vessel remodeling during the progression of $\mathrm{PH}$. Overproduction by P-ECs of FGF2 that acts on PA-SMCs appears to be a critical component of the abnormal crosstalk between these
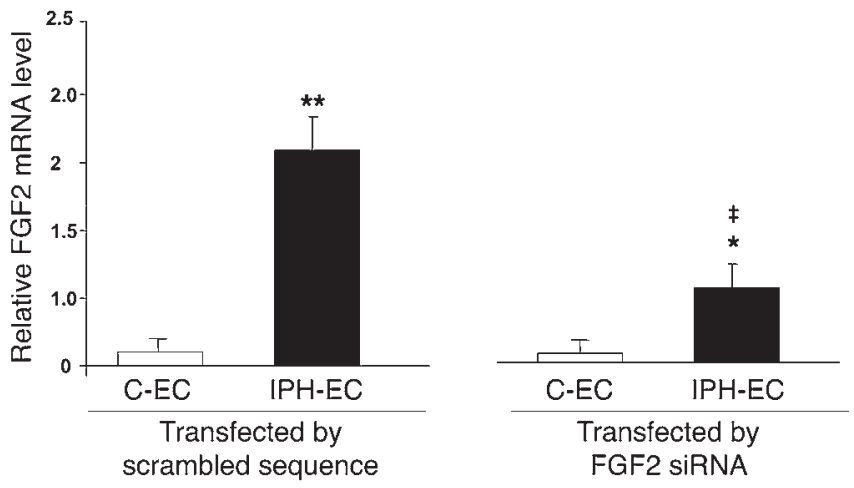

\section{Figure 2}

FGF2 mRNA level in P-ECs assessed by real-time quantitative PCR. FGF2 mRNA levels assessed by real-time quantitative PCR in P-ECs obtained from lungs of patients with IPH or controls and treated with FGF2-siRNA or the scrambled siRNA sequence. Values are mean \pm SEM from 5 controls and 5 patients with IPH. ${ }^{*} P<0.05$; ${ }^{*} P<0.001$ vs. $P$-ECs from control subjects; $¥ P<0.01$ between $P$-ECs treated with FGF2-siRNA vs. scrambled siRNA sequence. 

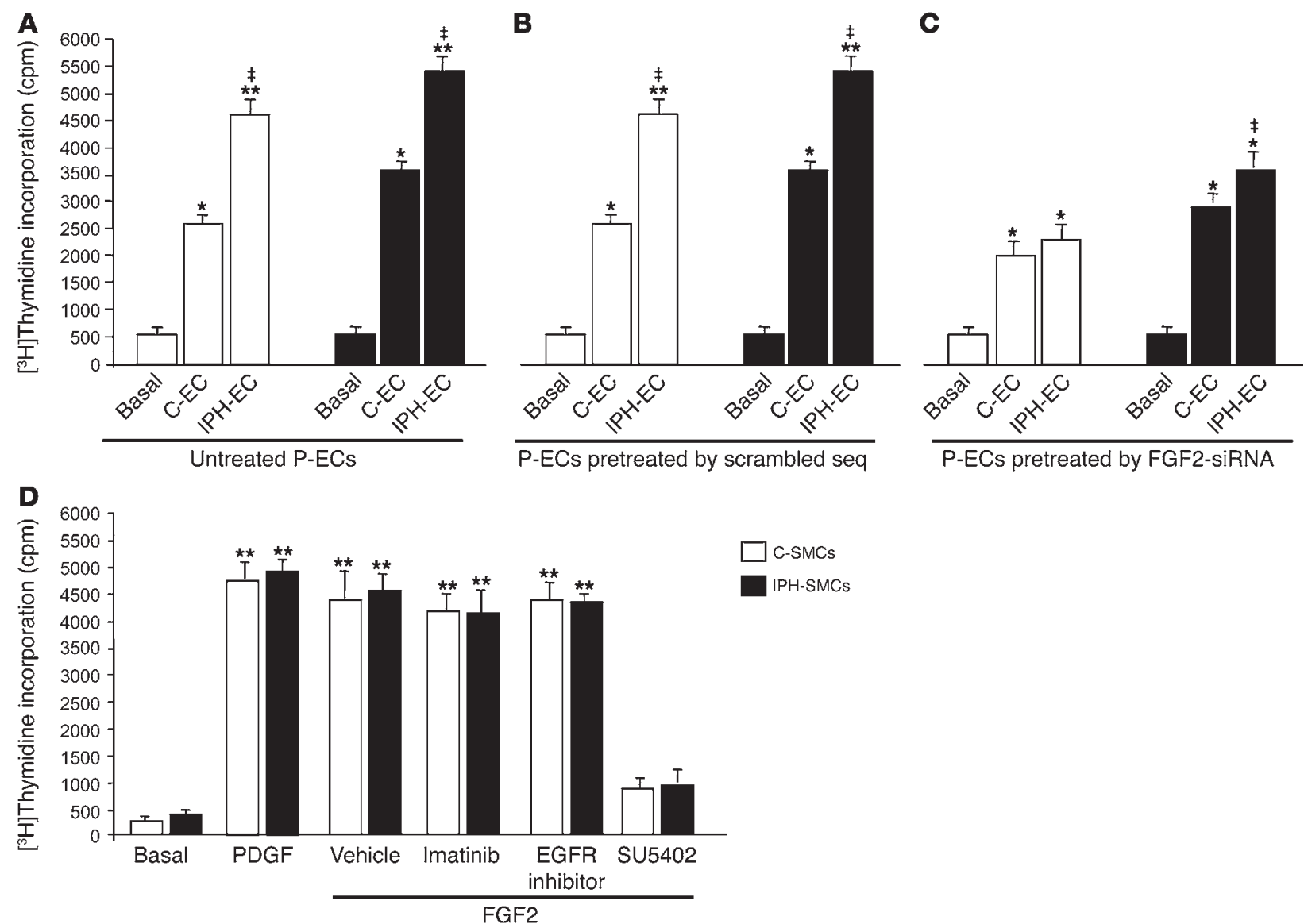

Figure 3

Effects of FGF2 release from P-ECs on PA-SMC growth. (A) Proliferation of PA-SMCs from controls and patients with IPH stimulated with medium of untreated P-ECs from controls and patients with IPH, (B) with medium from P-ECs pretreated with the scrambled siRNA sequence (seq), or (C) with medium from P-ECs pretreated with FGF2-siRNA. (D) Proliferation of PA-SMCs from controls and patients in response to PDGF $(10 \mathrm{ng} / \mathrm{ml})$ or to FGF2 $(10 \mathrm{ng} / \mathrm{ml})$ with or without the PDGFR antagonist imatinib, the EGFR inhibitor, or the FGFR1 antagonist SU5402 (10-5 M each). Values are mean \pm SEM of 4 experiments. ${ }^{*} P<0.01 ;{ }^{* *} P<0.001$ vs. basal conditions; $¥ P<0.01$ between stimulation with medium of P-ECs from IPH vs. P-ECs from controls.

2 cell types in human IPH. Using siRNA against human FGF2, we showed that endothelial FGF2 promoted PA-SMC growth and that this effect was stronger with P-ECs from IPH patients than with those from controls. The crucial role for endothelial FGF2 on $\mathrm{PH}$ development was further supported by experiments in rats with MCT-induced PH: FGF2-siRNA not only prevented the development of PH but also reversed established PH. Treatment with FGF2-siRNA reduced PA-SMC proliferation in situ, extracellular matrix accumulation, and perivascular inflammatory-cell infiltrates. Consistent with these findings, FGF pathway inhibition via FGFR1 blockade by the selective inhibitor SU5402 reversed MCTinduced PH. Taken together, these results suggest that dysregulation of FGF2 signaling may be a treatment target in $\mathrm{PH}$ in keeping with data obtained in studies of several malignant disorders.

In previous studies $(3,5)$, we found that excessive release of serotonin and ET-1, 2 soluble growth-promoting factors acting on PA-SMCs, was an intrinsic abnormality of P-ECs from patients with IPH. Although increased release of these mediators contributes to the exaggerated stimulating effect of P-EC medium from patients with IPH on PA-SMC growth compared with P-EC medium from controls, it is not the only source of this paracrine effect.
We measured growth factors in the medium of P-ECs from patients with IPH and from controls. Whereas the amounts of PDGF, EGF, and TGF- $\beta$ did not differ, the amount of FGF2 released by P-ECs from patients was 10 times greater than the amount released by P-ECs from controls. This difference was even more pronounced when the cells were incubated with human leukocyte elastase (data not shown) because FGF2 can be sequestered and stored in the extracellular matrix, then released by proteolytic processes (11-14, $25,26)$. Because the angiopoietin-1/Tie2 pathway is potentiated in IPH and contributes to PA-SMC hyperplasia via increased stimulation of serotonin and ET-1 synthesis by P-ECs (5), we investigated the effect of FGF2 on this pathway. Levels of mRNAs for ET-1 and Tie 2 were not significantly different between IPH P-ECs stimulated with exogenous FGF2 and IPH P-ECs stimulated with the vehicle (data not shown), suggesting that FGF2 did not affect or modulate the contribution of the angiopoietin-1/Tie2 pathway to the aberrant PA-SMC proliferation that characterizes the pathogenesis of $\mathrm{PH}$.

P-EC treatment with FGF2-siRNA induced $60 \%$ inhibition of the PA-SMC growth-promoting effect of P-EC medium from patients with IPH, whereas only $10 \%$ inhibition was observed with 


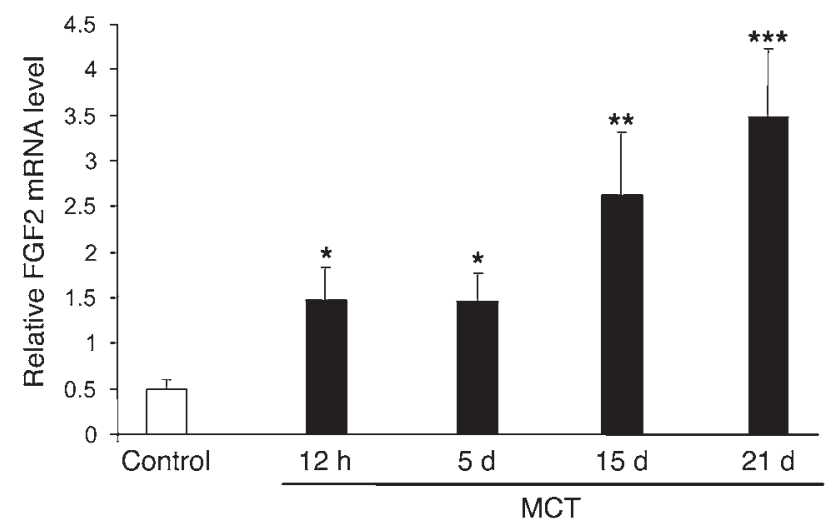

Figure 4

Lung FGF2 expression in MCT-induced PH. Kinetics of FGF2 mRNA levels measured by real-time quantitative $P C R$ over a 21-day period in lungs of rats ( $n=5$ in each group) injected s.c. with MCT $(60 \mathrm{mg} / \mathrm{kg})$. ${ }^{\star} P<0.05$; ${ }^{\star \star} P<0.01 ;{ }^{* \star} P<0.001$ vs. control rats injected with saline instead of MCT.

P-ECs from controls. Thus, our data suggest that dysregulation of the FGF2 signaling pathway may be related to overproduction of the ligand FGF2 but probably does not involve alteration of FGF2 receptor expression or signaling to PA-SMCs. Consistent with this hypothesis, PA-SMCs from patients with IPH stimulated by exogenous FGF2 did not grow faster than those from controls, and the selective FGFR tyrosine kinase inhibitor SU5402 completely abolished the FGF-induced proliferation of both groups of PA-SMCs. Moreover, the level of expression of FGFR1, the high-affinity FGFR for FGF2 on PA-SMCs, did not differ between patients and controls. Although autocrine FGF2 signaling was previously shown to induce mitosis and to inhibit apoptosis of SMCs $(27,28)$, we found evidence that the effect of FGF2 on PA-SMCs in IPH occurred chiefly via a paracrine mechanism. Indeed, only small amounts of FGF2 were detected in PA-SMC medium compared with P-EC medium, and FGF2-siRNA did not modify the response of PA-SMCs to exogenous FGF2, confirming the limited magnitude of autocrine FGF2 effects on PA-SMCs. Because activation of EGFR and PDGFR contributes to the PA-SMC proliferative response, we performed additional experiments to determine whether FGF signaling was related to activation of these receptors. Neither the selective PDGFR inhibitor (imatinib) nor the EGFR inhibitor affected FGF2-induced proliferation of PA-SMCs from controls or patients with IPH, suggesting no direct interaction between FGFR and PDGFR $\beta$ or EGFR signaling. Consistent with this finding, levels of mRNAs for PDGFR $\beta$ and EGFR were not significantly different between IPH PA-SMCs stimulated with exogenous FGF2 and IPH PA-SMCs stimulated with vehicle (data not shown).

Increased lung and circulating FGF2 levels have been reported in both experimental and human PH. Abnormally high levels of FGF2 have been reported in the blood of $51 \%$ and in the urine of $21 \%$ of patients with IPH (19) as well as in 2 animal models, a lamb model of $\mathrm{PH}$ developed by inserting an aortopulmonary vascular bypass graft (20) and the MCT-induced PH rat model (8). However, the importance of the FGF2 increase in initiating and perpetuating the disease was unclear. Increased FGF2 expression has also been documented in various malignancies in which
FGF2 acts on the tumor cells via either autocrine or paracrine mechanisms (29-32). In our study of patients with IPH, increased FGF2 expression was found in the lung and the FGF2 signal was predominantly located in the endothelium of pulmonary vessels as well as in cultured P-ECs removed from their abnormal in vivo environment. These results are reminiscent of those obtained using embryonic or cancer stem cells maintained in long-term cultures, which overexpress endogenous FGF2 (7). Thus, our present results strongly suggest that FGF2 overproduction may be closely linked to the abnormal P-EC phenotype in IPH and, therefore, to the pathogenesis of IPH.

To assess the in vivo effect of FGF2 on the development of $\mathrm{PH}$, we investigated the role of FGF2 in rats with experimental PH induced by MCT injection. When administered s.c., MCT is converted to its pneumotoxic metabolites in the liver; these are transported to the lungs, where they cause early injury to the pulmonary arterial endothelium and an inflammatory response before the onset of PA-SMC proliferation and the development of $\mathrm{PH}$ $(21-24,33,34)$. Alterations in the structure of the pulmonary vessel walls include (a) thickening of the medial layer of the SMCs, (b) increased extracellular matrix synthesis and deposition, and (c) increased thickness of the adventitial layer because of edema and mononuclear inflammatory cell infiltrates composed mainly of macrophages (21-24). This increased pulmonary vascular remodeling causes an increase in PAP that eventually leads to RV hypertrophy. Morbidity and mortality in MCT-treated rats are known to be linked to vascular disease (24). Although the vascular changes that occur in response to MCT injection in rats do not resemble the complex pulmonary vascular lesions seen in patients with primary or advanced secondary $\mathrm{PH}$, our results support a pathophysiological role for FGF2 in the development of PH, where FGF2 may serve as a link between altered EC signaling and pulmonary vascular remodeling. First, as previously described (8), we found that lung FGF2 expression increased markedly during the development of MCT-induced PH and that this increase was detected as early as 12 hours after injection at a time when PA-SMC proliferation was not yet detectable. Second, we found that pretreatment of MCT-injected rats with FGF2-siRNA before the MCT injection was followed by marked attenuation of $\mathrm{PH}$ development, as assessed on day 21 based on decreases in PAP, RV hypertrophy, and distal pulmonary artery muscularization, compared with pretreatment of MCT-injected rats with the scrambled siRNA sequence. These data constitute strong evidence that upregulation of lung FGF2 in response to MCT contributes to the initiation of PA-SMC proliferation and subsequent development of $\mathrm{PH}$. Treatment of rats with FGF2-siRNA prior to MCT injection prevented the early rise in FGF2, and repeated administration of FGF2-siRNA 24 hours after MCT not only prevented the increase but also induced a major decrease in FGF2 mRNA and protein levels on day 5. In contrast, in the heart and liver, FGF2-siRNA did not significantly affect FGF2 mRNA or protein levels compared with control siRNA (data not shown). Third, we found that treatment with FGF2-siRNA not only prevented but also almost completely reversed established $\mathrm{PH}$, suggesting that a sustained increase in lung FGF2 expression may be necessary for both the progression and the maintenance of MCT-induced PH. Consistent with this finding, blocking FGF2 signaling by the selective FGFR inhibitor SU5402 reversed established MCT-induced PH in rats, decreasing the values of PAP, RV hypertrophy, and peripheral artery muscularization to the levels observed in vehicle-treated rats. 

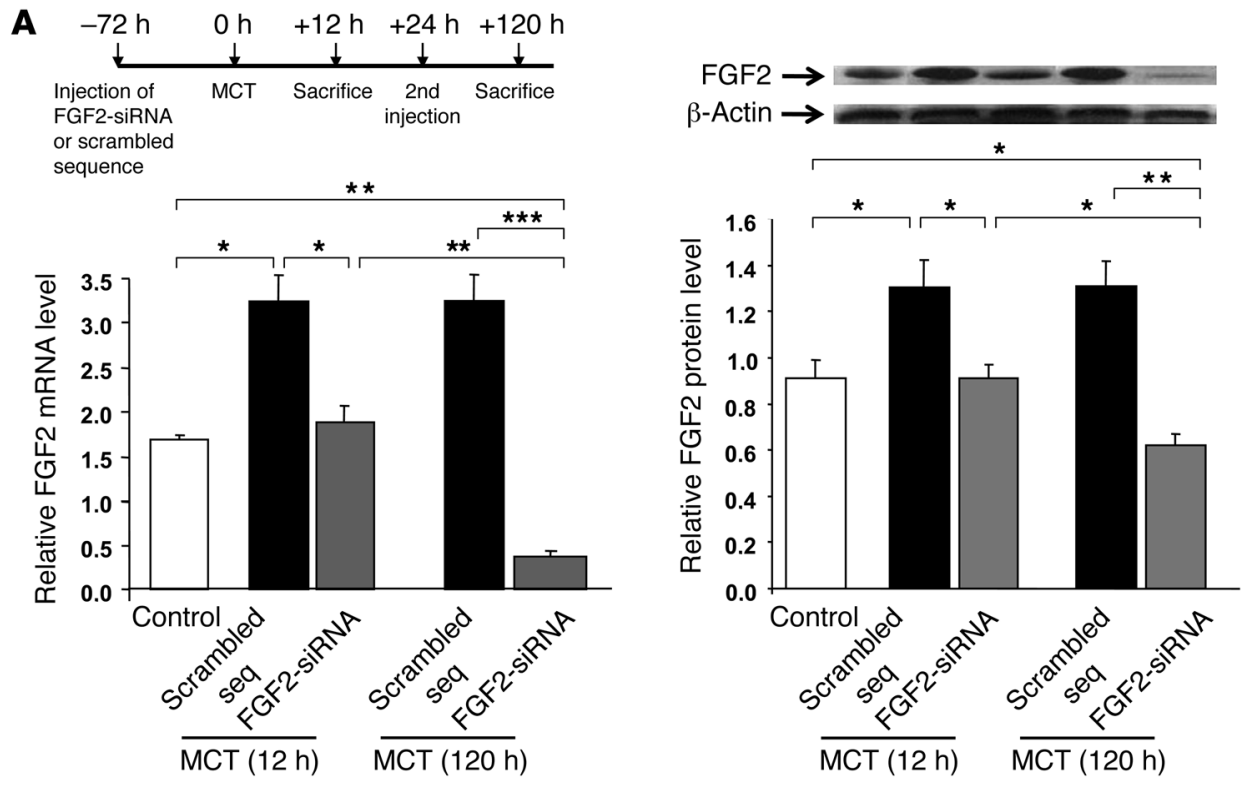

Figure 5

Validation of acute and chronic lung FGF2 knockdown by siRNA. (A) FGF2 mRNA and protein levels in rat lung 12 hours and 120 hours after MCT injection. Rats were injected with FGF2-siRNA or the scrambled siRNA sequence either once, 3 days before MCT injection (12-hour group), or twice, 3 days before and 1 day after MCT injection (120-hour group). (B) FGF2 mRNA and protein levels in rat lungs 21 days after MCT injection. Rats were injected with FGF2-siRNA or the scrambled siRNA sequence (2 [2iv] or 4 [4iv] injections). FGF2 mRNA levels were assessed using real-time quantitative PCR and protein levels with FGF2 immunoblotting (the lanes were run on the same gel but were not contiguous). All values are mean \pm SEM from at least 5 animals in each group. ${ }^{\star} P<0.05 ;{ }^{* \star} P<0.01 ;{ }^{* \star *} P<0.001$
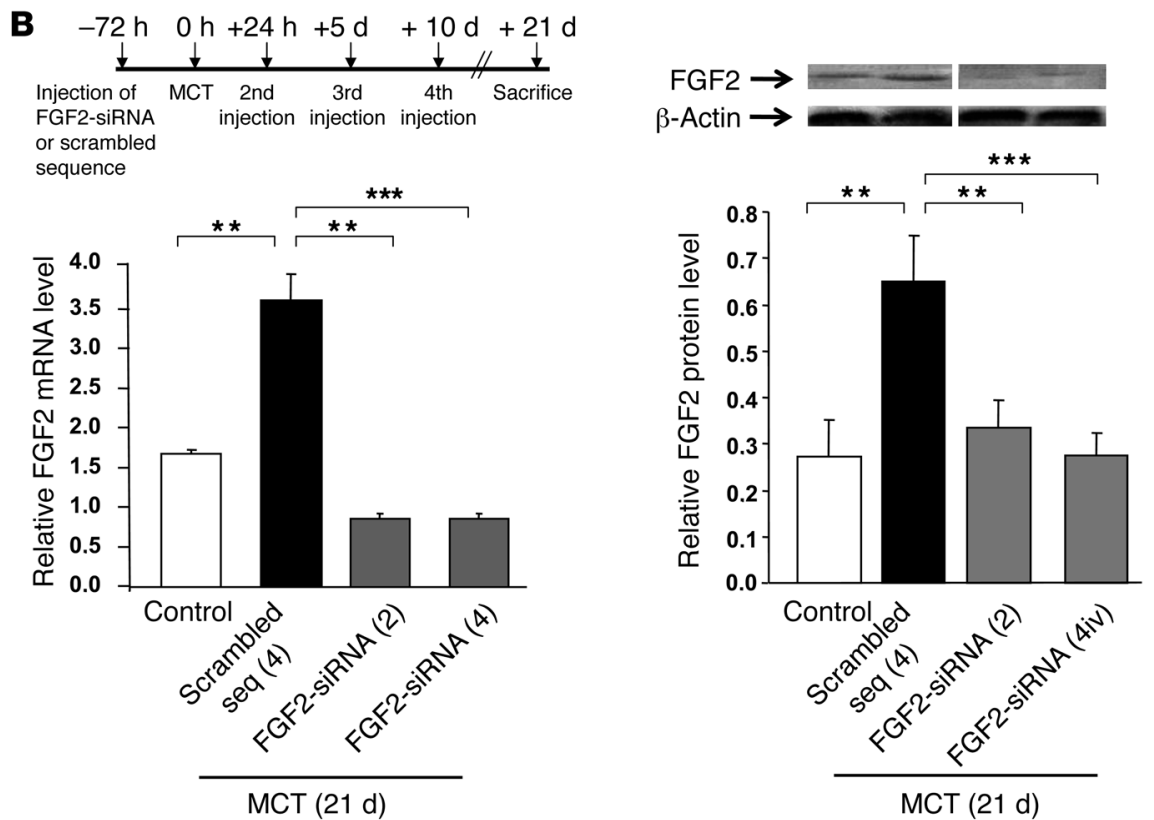
vs. rats injected with saline instead of MCT or vs. rats treated with the scrambled siRNA sequence.

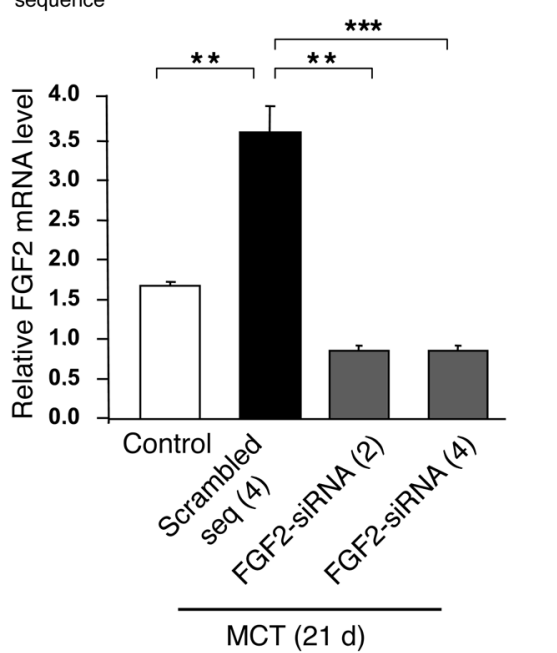

Because PA-SMC hyperplasia is among the main pathological changes in patients with $\mathrm{PH}$, we focused on the growth-promoting effect of FGF2 on PA-SMCs and found strong inhibition of SMC proliferation by FGF pathway blockade with either FGF2-siRNA or SU5402. However, we cannot exclude the possibility that FGF blockade acts via a synergistic effect on apoptosis. Pardo et al. reported that FGF2 increased the expression of the antiapoptotic proteins XIAP and Bcl-XL through the PKCE signaling pathway to inhibit apoptosis of lung cancer cells (35). Moreover, Xiao et al. reported that FGF2 inhibited apoptosis in human small-cell lung cancer cells via upregulation of the survivin protein and decreased release of Smac from mitochondria to the cytoplasm (36). Moreover, the multifunctional role for the FGF signaling pathway may not be confined to direct effects on PA-SMCs during $\mathrm{PH}$ progression. FGF2 not only increases PA-SMC prolifera- tion but also directly modulates proteolytic processes via effects on the MMPs and urokinase-type plasminogen activator/plasmin systems, e.g., via regulation of the expression of collagenase (37), matrilysin (38), stromelysin-1 (39), and the urokinase-type plasminogen activator $(40,41)$, which contribute both to pulmonary vascular remodeling and to the release of growth factors such as FGF2 from the extracellular matrix. FGF2 also stimulates the expression of adhesion molecules on ECs, thereby promoting the recruitment of inflammatory cells and potentiating the inflammatory process (42). In addition to FGFR, activation of 2 other tyrosine kinase receptors, EGFR and PDGFR $\beta$, have been implicated in the pathogenesis of $\mathrm{PH}(43,44)$ : Merklinger et al. reported that selective blockade of EGFR signaling induces regression of medial hypertrophy through SMC apoptosis and reverses progressive PH (43), while Schermuly et al. reported that the PDGF 


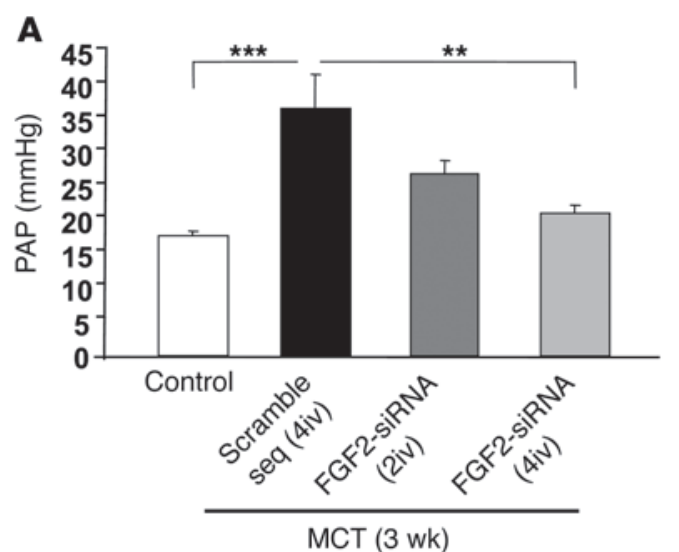

C
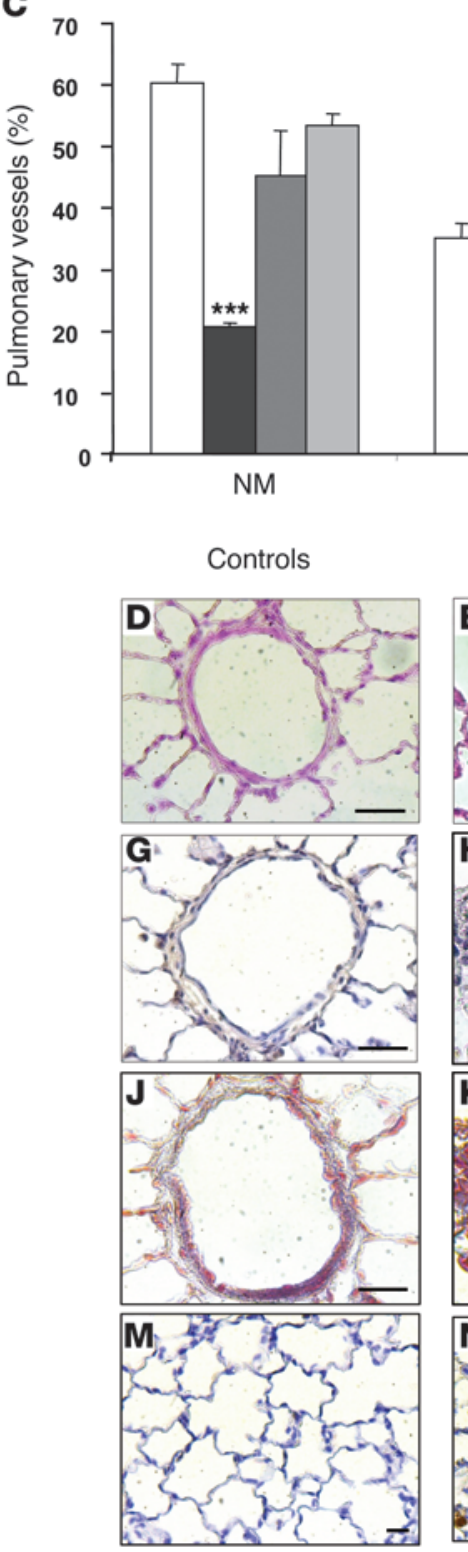

B

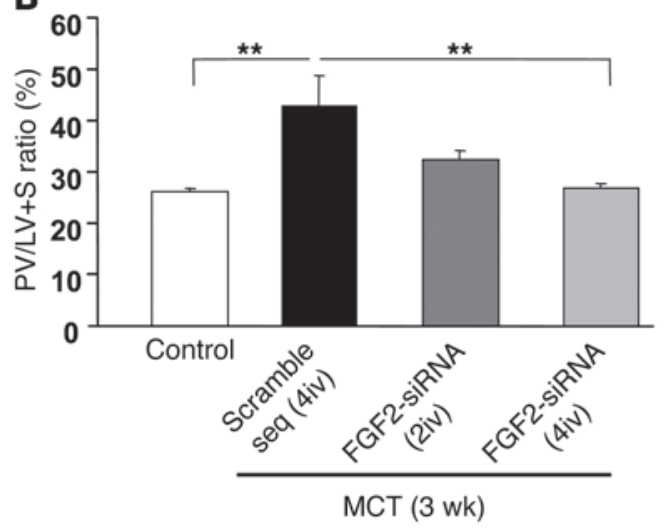

Figure 6

Evaluation of the efficacy of FGF2-siRNA pretreatment in MCT-induced $\mathrm{PH}$. Results obtained in rats treated with FGF2-siRNA or the scrambled siRNA sequence before the MCT injection and studied on day 21. Compared with the scrambled siRNA sequence, FGF2-siRNA significantly prevented the development of $\mathrm{PH}$, as assessed by (A) PAP; (B) RV hypertrophy reflected by the $R V /(L V+S)$ weight ratio; and $(\mathbf{C})$ percentages of $\mathrm{NM}, \mathrm{PM}, \mathrm{FM}$, and FM+ intraacinar vessels. (D-F) Hematoxylin-phloxine-saffron stain; (G-I) PCNA immunostaining; (J-L) Masson trichrome stain; and (M-O) CD68 immunostaining of rat lungs. ${ }^{\star} P<0.05$; ${ }^{* \star} P<0.01$; ${ }^{\star \star \star} P<0.001$ vs. rats injected with saline instead of MCT or vs. rats treated with scrambled siRNA sequence. Scale bars: $25 \mu \mathrm{m}$. 


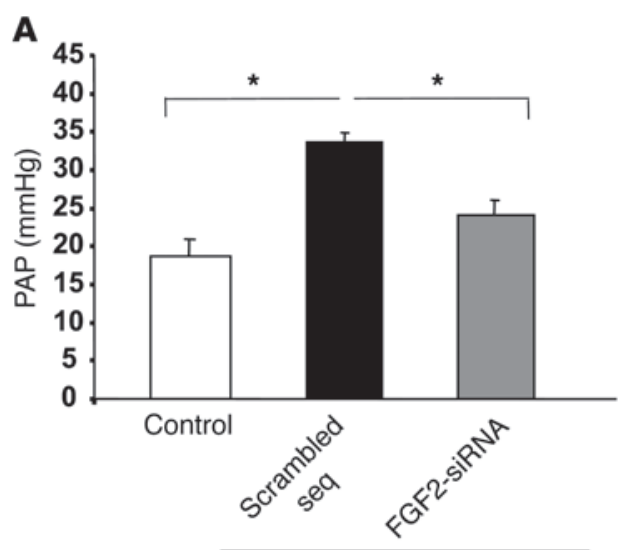

MCT (6 wk)
B

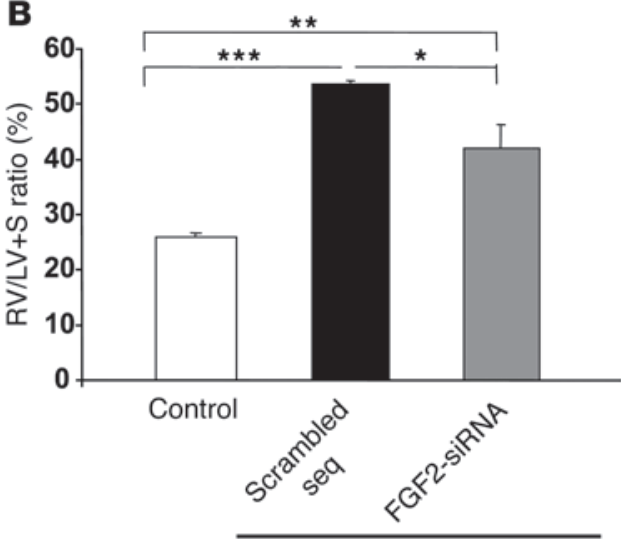

MCT (6 wk)

\section{C}

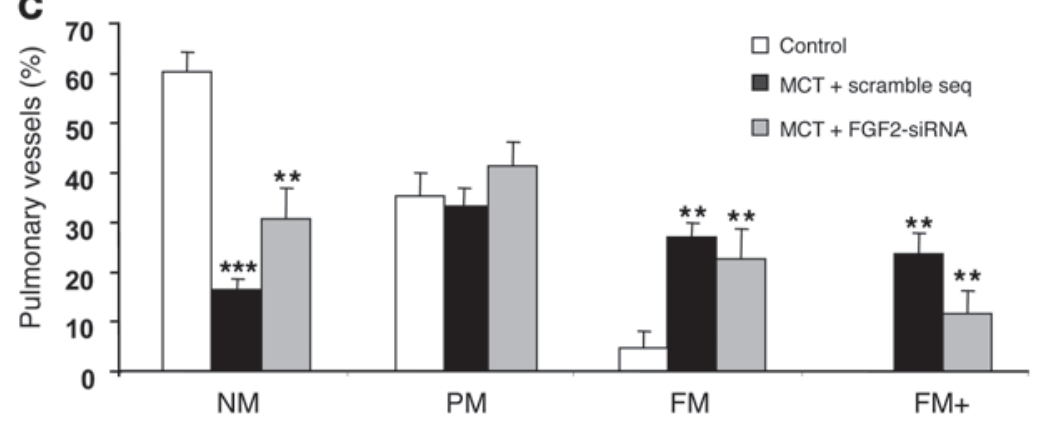

6 wk MCT + scramble siRNA
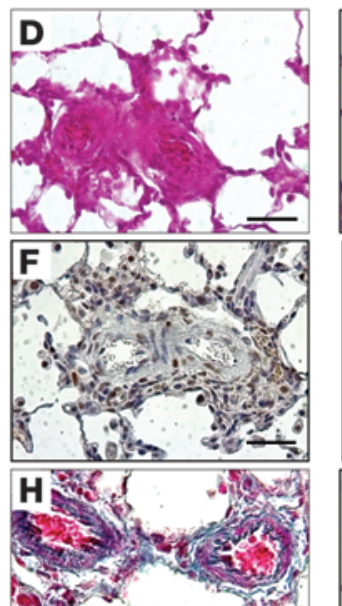

Pige

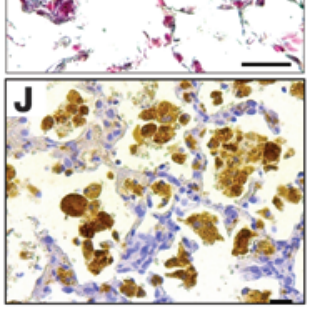

6 wk MCT +

FGF2-siRNA
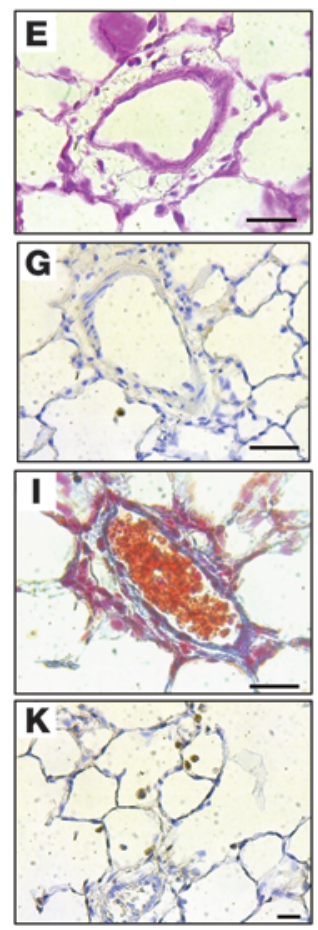

\section{Figure 7}

Evaluation of the efficacy of FGF2siRNA treatment in MCT-induced $\mathrm{PH}$. Results obtained in rats treated with FGF2-siRNA or the scrambled siRNA sequence (2 injections) on day 21 and day 25 after MCT injection (treatment) and studied on day 42. Compared with the scrambled siRNA sequence, FGF2-siRNA significantly reversed the development of $\mathrm{PH}$, as assessed by (A) PAP; (B) RV hypertrophy reflected by the $\mathrm{RV} /(\mathrm{LV}+\mathrm{S})$ weight ratio; and (C) percentages of NM, PM, FM, and $\mathrm{FM}+$ intra-acinar vessels. (D and $\mathbf{E}$ ) Hematoxylin-phloxine-saffron stain; ( $\mathbf{F}$ and $\mathbf{G}$ ) PCNA immunostaining; ( $\mathbf{H}$ and I) Masson trichrome stain; and ( $\mathbf{J}$ and $\mathbf{K}$ ) CD68 immunostaining of rat lungs. ${ }^{*} P<0.05$; ${ }^{* \star} P<0.01$; ${ }^{* * *} P<0.001$ vs. rats injected with saline instead of MCT or vs. rats treated with scrambled siRNA sequence. Scale bars: $25 \mu \mathrm{m}$. 

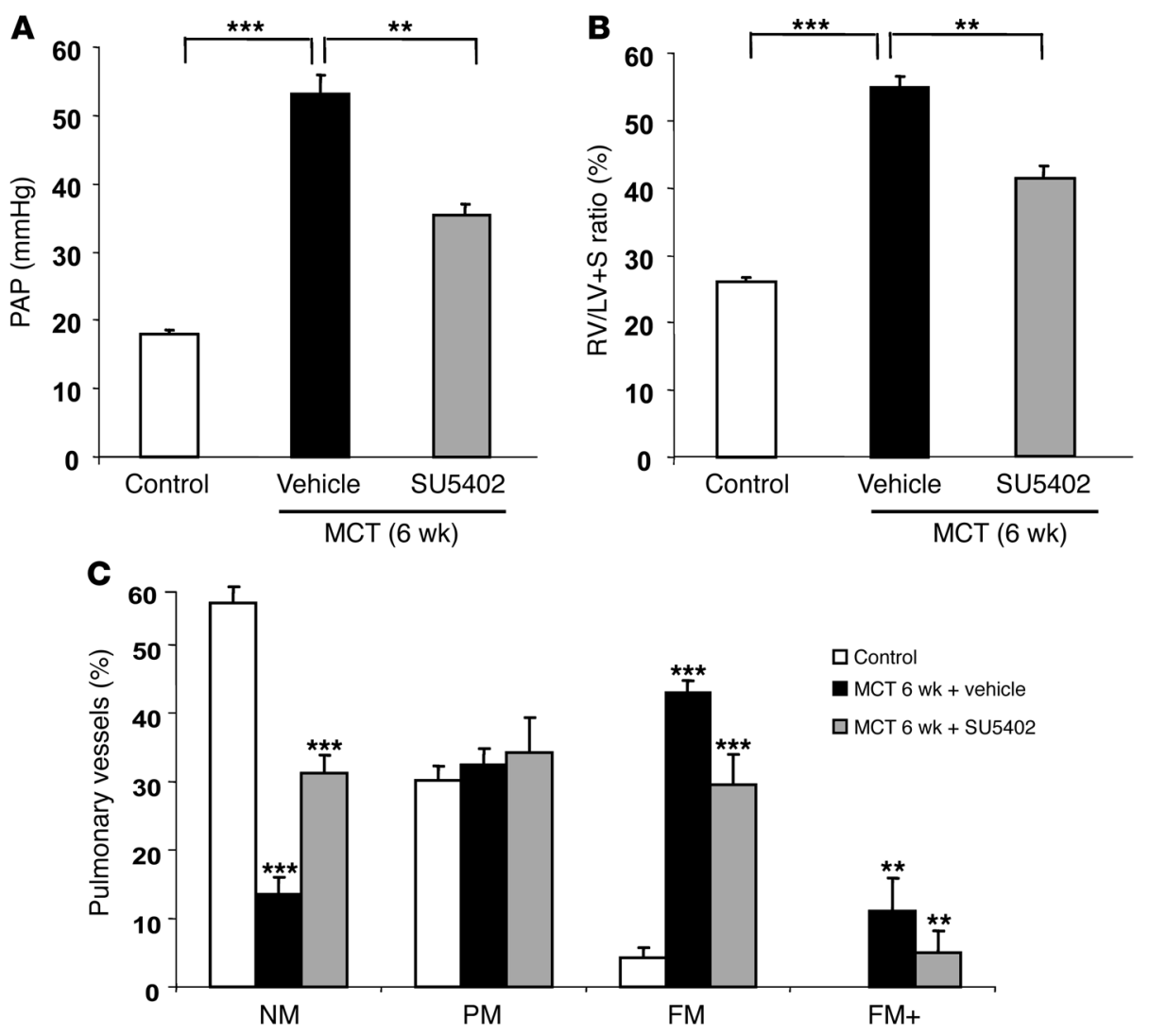

Figure 8

Reversal of MCT-induced $\mathrm{PH}$ with the FGFR1 inhibitor SU5402. Results obtained in rats treated with SU5402 $\left(25 \mathrm{mg} \times \mathrm{kg}^{-1} \times \mathrm{d}^{-1}\right.$ ) or vehicle (saline) from day 21 to day 42 after MCT injection (treatment) and studied on day 42. Compared with vehicle, SU5402 significantly reversed the development of $\mathrm{PH}$, as assessed by (A) PAP; (B) RV hypertrophy reflected by the $R V /(L V+S)$ weight ratio; and (C) percentages of NM, PM, FM, and $\mathrm{FM}+$ intra-acinar vessels. ${ }^{* *} P<0.01$; ${ }^{* \star *} P<0.001$ vs. rats injected with saline instead of MCT vs. rats treated with saline instead of SU5402. receptor antagonist imatinib mesylate reverses pulmonary vascular remodeling in 2 different animal models of PH (44). In the present study, we did not observe a direct relationship between FGF2 and the expression or activation of EGFR or PDGFR $\beta$, but it is possible that these key elements affect common critical mediators of vascular remodeling in similar ways.

\section{Methods}

Patients with pulmonary arterial hypertension and controls. We studied lung specimens obtained during lung transplantation in 8 patients with IPH and during lobectomy or pneumonectomy for localized lung cancer in 8 controls. Age (mean $\pm \mathrm{SD}$ ) was $34 \pm 10$ years in the patients with IPH and $56 \pm 12$ in the controls. Mean PAP in the patients with IPH was $66 \pm 17 \mathrm{mmHg}$. Preoperative echocardiography was performed in the controls to rule out $\mathrm{PH}$, and the lung specimens from the controls were collected at a distance from tumor foci. This study was approved by the local ethics committee (CPP Ile-de-France VII, Le Kremlin-Bicêtre, France), and patients provided informed consent prior to their contribution to the study.

Isolation and culture of P-ECs and PA-SMCs. Human P-ECs and PA-SMCs were isolated and cultured as previously described $(3,45)$. To characterize the endothelial cell phenotype, P-ECs were labeled with acetylated lowdensity lipoprotein coupled to a fluorescent carbocyanine dye (1,1'-dioctadecyl-3,3,3', $3^{\prime}$-tetramethylindocarbocyanine perchlorate [DiI-Ac-LDL]) and stained with antibodies against the endothelial cell-specific Ulex europaeus agglutinin-1 (UEA-1) Cells positive for DiI-Ac-LDL and UEA-1 and negative for desmin and vimentin were classified as P-ECs and contributed more than $95 \%$ of the cell-culture population. To identify PA-SMCs, we examined cultured cells for expression of muscle-specific contractile and cytoskeletal proteins including smooth-muscle $\alpha$-actin, desmin, and vinculin. Cells were used between passages 3 and 6 .
Measurements of production of FGF2 and other growth factors by P-ECs. To quantify SMC growth factors in P-EC culture medium, we seeded P-ECs from controls and patients with IPH on 6-well plates at a density of $3 \times 10^{5}$ cells/ well and allowed the cells to adhere. After 24 hours, the cells were incubated in serum-free MCDB131 for 24 hours. PDGF-BB, TGF- $\beta 1$, FGF2, and EGF were measured in the P-EC culture medium using ELISAs (R\&D Systems).

FGF2 knockdown in P-ECs by siRNA. To determine whether FGF2 produced by P-ECs affected PA-SMC proliferation, we seeded PA-ECs from controls and patients with IPH, then grew them to $70 \%$ confluence in medium supplemented with $10 \%$ FCS. Transfection with $50 \mathrm{nM}$ of FGF2siRNA or scramble sequence (control siRNA) was achieved using Lipofectamine 2000 transfection reagent (Invitrogen) according to the manufacturer's protocol. The transfected cells were incubated for 48 hours in medium supplemented with $15 \%$ FCS, then maintained for 24 hours in serum-free medium. The media were collected for treatment of PA-SMCs, whereas the cells were harvested for measurement of FGF2 mRNA. The siRNA duplex sequences used to target FGF2 (FGF2-siRNA) were as follows: sense, 5'-GCACUGAAACGAACUGGGCAGUAU-3'; and antisense, $5^{\prime}$-UAUACUGCCCAGUUCGUUUCAGUGC- ${ }^{\prime}$. The scramble sequences were as follows: sense, 5'-GCAAGAAGCAAUCGGCGGAUUCAUA-3'; and antisense 5'-AUGAAUCCGCCGAUUGCUUCUUGC-3'.

SMC proliferation assessed by $\left[{ }^{3} H\right]$ thymidine incorporation. PA-SMCs in DMEM supplemented with $15 \%$ FCS were seeded in 24 -well plates at a density of $5 \times 10^{4}$ cells/well and allowed to adhere. The cells were subjected to 48 hours of growth arrest in serum-free medium, then treated with $1 \mathrm{ml}$ of conditioned P-EC medium. We also tested the effect of exogenous PDGF $(10 \mathrm{ng} / \mathrm{ml})$ and FGF2 $(10 \mathrm{ng} / \mathrm{ml})$ on PA-SMC proliferation with or without imatinib $\left(10^{-5} \mathrm{M}\right)$, EGF antagonist $\left(10^{-5} \mathrm{M} ; 324674\right.$, Calbiochem), and SU5402 (10-5 M; 572630, Calbiochem). Under each condition, $\left[{ }^{3} \mathrm{H}\right]$ thymidine $(1 \mu \mathrm{Ci} / \mathrm{ml})$ was added to each well. After incubation for 
24 hours, the cells were washed twice with PBS, treated with ice-cold $10 \%$ trichloroacetic acid, and dissolved in $0.1 \mathrm{~N} \mathrm{NaOH}(0.5 \mathrm{ml} /$ well). The incorporated radioactivity was counted and reported as $\mathrm{cpm} /$ well.

Real-time quantitative RT-PCR for measurement of FGF2 and FGFR1 mRNA levels. FGF2 expression was examined in lungs and P-ECs from patients with IPH and from controls. Levels of FGF2 mRNA were measured also in rat lungs at various times between day 1 and day 21 after the MCT injection. Finally, FGFR1 expression was assessed in PA-SMCs from patients with IPH and from controls.

Total RNA was extracted using the QIAGEN RNeasy Mini Kit (QIAGEN) according to the manufacturer's instructions. RNA concentrations were determined using standard spectrophotometric techniques, and RNA integrity was assessed by visual inspection of ethidium bromidestained agarose gels.

Reverse transcription was performed using random hexamer primers and reverse transcriptase (Invitrogen). For PCR, we used Primer Express Software, version 2.0 (Applied Biosystems) to design a sense primer (5'-ACGGCGTCCGGGAGAA-3') and an antisense primer $\left(5^{\prime}\right.$ ACACTCCCTTGATGGACACAACT- $3^{\prime}$ ) for FGF2 and a sense primer (5'-TCTTCTGGTTCGGCATCAC-3') and an antisense primer (5'-TTCGGGAAGCTCATACTCAGAGA-3') for FGFR1. To avoid inappropriate amplification of residual genomic DNA, intron-spanning primers were selected and internal control $18 \mathrm{~S}$ rRNA primers provided. For each sample, the amplification reaction was performed in duplicate using SYBR Green Mix (Applied Biosystems) and specific primers. Signal detection and result analysis were achieved using ABI PRISM 7000 sequence detection software (Applied Biosystems). Expression of the gene of interest was computed relative to the expression of the internal standard r18S, as follows: $\mathrm{mRNA}=1 / 2^{\text {(Ct gene of interest }-\mathrm{Ct} \text { r18s) }}$.

In situ bybridization. In situ hybridization was performed on $5-\mu \mathrm{m}$ thick sections of lungs from patients with IPH and from controls, using $3^{\prime}-\left[{ }^{35} \mathrm{~S}\right]-$ labeled oligonucleotide probes specific for human FGF2. Terminal deoxynucleotidyl transferase (TdT) was used to label $1 \mathrm{pmol}$ of oligonucleotide probe in $1 \times \mathrm{TdT}$ buffer (GE Healthcare) with a 20 -fold excess $\left[{ }^{35} \mathrm{~S}\right] \mathrm{dATP}$ (PerkinElmer) for 1 hour at $37^{\circ} \mathrm{C}$. Probes were purified with the QIAquick Nucleotide Removal Kit (QIAGEN). Sections were equilibrated at room temperature, then covered with $200 \mu$ l of hybridization mixture containing the $\left[{ }^{35} \mathrm{~S}\right]$-labeled probe $\left(10^{4} \mathrm{cpm} / \mu \mathrm{l}\right)$ diluted in hybridization buffer. Hybridization was then allowed to occur overnight in a moist chamber at $42^{\circ} \mathrm{C}$. slides were rinsed in sodium chloride/sodium citrate ( $150 \mathrm{mM} \mathrm{NaCl}, 15 \mathrm{mM}$ $\mathrm{Na}_{3} \mathrm{C}_{6} \mathrm{H}_{5} \mathrm{O}_{7}, \mathrm{pH}$ 7.0), dehydrated, and dried, as described elsewhere (46). Sections hybridized to the sense probe served as the negative control. Sections were exposed to film as described above and developed after 7-14 days.

The sections were finally dipped in Kodak NTB2 nuclear track emulsion at $37^{\circ} \mathrm{C}$ and dried in complete darkness at room temperature. The slides were stored at $4^{\circ} \mathrm{C}$ until developed in Kodak D19 and fixed in Kodak 3000A. To visualize the cells, we used weak counterstaining with hematoxylin blue.

Western blot assay. Tissue samples from rat lungs were homogenized in cold PBS containing protease inhibitors, and the protein concentration was determined using the Bradford protein assay (Bio-Rad). Samples containing $100 \mu \mathrm{g}$ proteins were fractionated by $12 \%$ sodium dodecyl sulfate polyacrylamide gel electrophoresis and transferred to nitrocellulose membranes. FGF2 was then detected using a rabbit anti-FGF2 polyclonal antibody (Chemicon) diluted 1:300 in 1\% milk. The second antibody was a polyclonal anti-rabbit antibody diluted 1:5000 (Santa Cruz Biotechnology Inc.). Immunoreactive bands were visualized using chemiluminescence (ECL) (GE Healthcare). A polyclonal antibody against $\beta$-actin (diluted 1:3000; Sigma-Aldrich) served as the internal control. Densitometric quantification of the immunoblot bands was performed using semiautomated image analysis (NIH Image 1.52), as previously described (33).
Enzyme-linked immunosorbent assay. Lung lysates and vascular-cell culture medium from patients and controls were used for this experiment. In brief, $100 \mu \mathrm{l}$ of cell or lung supernatant was incubated with $50 \mu \mathrm{l}$ of assay diluent for 2 hours at room temperature in a 96-well plate coated with a monoclonal antibody against FGF2. After 3 washes, a conjugate of polyclonal FGF2 antibody and horseradish peroxidase was added and incubated for 2 hours at room temperature. After addition of a substrate solution and incubation for 30 minutes, stop solution was added, and absorbance was measured at $450 \mathrm{~nm}$ in a ThermoMax Microplate Reader (Molecular Devices).

Experimental $\mathrm{PH}$ in rats. $\mathrm{PH}$ was induced in rats by a single s.c. injection of MCT (60 mg/kg) (33). Adult male Wistar rats weighing 200-250 g were used (Charles River). Animal experiments performed in this study were approved by the Institutional Animal Care and Use Committee of the Faculté de Médecine and the French National Institute of Health and Medical Research, Créteil, France).

Assessment of $\mathrm{PH}$. Rats were anesthetized with sodium pentobarbital $(60 \mathrm{mg} / \mathrm{kg}$, i.p.). A polyvinyl catheter was introduced into the right jugular vein, then pushed through the RV into the pulmonary artery. A polyethylene catheter was inserted into the right carotid artery. PAP and systemic artery pressure were measured, the thorax was opened, and the left lung was immediately removed and frozen in liquid nitrogen. The heart was dissected and weighed for calculation of the RV hypertrophy index $(\mathrm{RV} /[\mathrm{LV}+\mathrm{S}])$. The right lung was fixed in the distended state with formalin buffer. After routine processing and paraffin embedding, multiple sections from each lobe were stained with H\&E. In each rat, 60 intra-acinar arteries were examined and categorized as nonmuscular (NM), partially muscular (PM), fully muscular (FM), or obliterated (FM+).

Validation of siRNA efficiency in achieving FGF2 knockdown in lungs from MCTinjected rats. To assess the effect of siRNA targeting FGF2 on the expression of the gene, we used 2 protocols. In protocol 1, FGF2-siRNA $(0.5 \mathrm{nmol} / 100 \mu \mathrm{l})$ was given i.v. 3 days before and 1 day after the MCT injection. In protocol 2, 2 siRNA injections were given as in protocol 1 and followed by 2 further injections, on day 5 and day 10 , respectively. The animals were killed 12 hours, 120 hours, and 21 days after the MCT injection. The lungs and hearts were removed, the RNA extracted, and the FGF2 mRNA levels assayed using quantitative real-time PCR.

Effects of FGF2 knockdown before and after the development of MCT-induced $\mathrm{PH}$ in rats. To assess the potential effects of FGF2 knockdown used before and after the development of $\mathrm{PH}$ induced by MCT $(60 \mathrm{mg} / \mathrm{kg}$ s.c.), we randomly divided 50 rats into 5 groups of 10 animals. In the pretreatment protocol, 1 group received 2 i.v. FGF2-siRNA injections according to protocol 1, 1 group received 4 injections according to protocol 2 , and 1 group received control siRNA injections at the same times as in protocol 2. In the treatment, the rats were left untreated for 21 days, then divided randomly into 2 groups, of which one received FGF2-siRNA and the other received control siRNA. The FGF2-siRNA injections were given on day 21 and day 25 after the MCT injection.

Effect of treatment with SU5402 on established MCT PH. To assess the potential effects of the FGFR1 inhibitor SU5402 on established PH, adult male Wistar rats (200-250 g) were given MCT ( $60 \mathrm{mg} / \mathrm{kg}$ s.c.), left untreated for 21 days, then randomly divided into 2 groups (10 animals in each group), of which one was treated with SU5402 $(25 \mathrm{mg} / \mathrm{kg} / \mathrm{day})$ and the other given the vehicle, from day 21 to day 42 . All treatments were given once a day by s.c. injection (47).

In situ SMC proliferation, matrix accumulation, and inflammation. To determine the mechanism by which FGF2-siRNA diminished PH induced by MCT, PCNA labeling, collagen accumulation, and infiltrating macrophages were measured in rat lung sections. Tissue sections were deparaffinized in xylene, then treated with a graded series of alcohol washes, rehydrated in PBS ( $\mathrm{pH} 7.5$ ), and incubated with target retrieval solution (Dako) in a water 
bath at $90^{\circ} \mathrm{C}$ for 20 minutes. Endogenous peroxidase activity was blocked with $\mathrm{H}_{2} \mathrm{O}_{2}$ in $\mathrm{PBS}(3 \%, \mathrm{v} / \mathrm{v})$ for 5 minutes. Slides were washed with PBS, incubated for 30 minutes in a protein-blocking solution, and incubated for 30 minutes with anti-PCNA mouse monoclonal antibody (PC-10, 1:200; Dako) or anti-CD68 antibody (Hycult Biotechnology) diluted 1:200. Then the slides were processed using the alkaline phosphatase LSAB+ system horseradish peroxidase detection kit (Dako). Brown color was generated by a diaminobenzidine substrate, and nuclei were counterstained with hematoxylin. Slides were stained with the collagen-specific Masson trichrome dye (48). Image analysis was performed with a charge-coupled device Iris camera (CDD Iris; Sony) coupled with a light microscope (Laborlux).

Statistics. Data are expressed as mean \pm SEM. The nonparametric MannWhitney test was used for comparisons between 2 groups. Comparisons of data obtained at various times after MCT injection or in various treatment groups were performed using the nonparametric Kruskal-Wallis test followed, if significant, by Dunn's test. To compare the degree of pulmonary arterial muscularization between groups, we used the nonparametric Mann-Whitney or Kruskal-Wallis test after ordinal classification of the vessels as $\mathrm{NM}, \mathrm{PM}, \mathrm{FM}$, or FM+. The effect of siRNA injection on FGF2 expression in the lung at various times after MCT injection was evaluated by 2 -way ANOVA, testing for treatment and time effects. When a time-bytreatment interaction was found, vehicle and active treatment were compared using the nonparametric Mann-Whitney test.

\section{Acknowledgments}

This study was supported by grants from the Leg Poix, INSERM, Ministère de la Recherche, and Institut des Maladies Rares (GIS). This publication reflects only the authors' views, and under no circumstances is the European Community liable for any use that may be made of the information it contains.

Received for publication January 18, 2008, and accepted in revised form December 22, 2008.

Address correspondence to: Saadia Eddahibi, INSERM U841, Faculté de Médecine, 8 avenue du Général Sarrail, 94010 Créteil, France. Phone: 33-149-813-613; Fax: 33-148-981-777; E-mail: saadia.eddahibi@inserm.fr.
1. Rubin, L.J. 1997. Primary pulmonary hypertension. N. Engl.J. Med. 336:111-117.

2. Humbert, M., et al. 2004. Cellular and molecular pathobiology of pulmonary arterial hypertension. J. Am. Coll. Cardiol. 43:13S-24S.

3. Eddahibi, S., et al. 2006. Cross talk between endothelial and smooth muscle cells in pulmonary hypertension: critical role for serotonininduced smooth muscle hyperplasia. Circulation. 113:1857-1864.

4. Armulik, A., Abramsson, A., and Betsholtz, C. 2005. Endothelial/pericyte interactions. Circ. Res. 97:512-523.

5. Dewachter, L., et al. 2006. Angiopoietin/Tie2 pathway influences smooth muscle hyperplasia in idiopathic pulmonary hypertension. Am. J. Respir. Crit. Care Med. 174:1025-1033.

6. Powers, C.J., McLeskey, S.W., and Wellstein, A. 2000. Fibroblast growth factors, their receptors and signaling. Endocr. Relat. Cancer. 7:165-197.

7. Presta, M., et al. 2005. Fibroblast growth factor/ fibroblast growth factor receptor system in angiogenesis. Cytokine Growth Factor Rev. 16:159-178.

8. Arcot, S.S., Fagerland, J.A., Lipke, D.W., Gillespie, M.N., and Olson, J.W. 1995. Basic fibroblast growth factor alterations during development of monocrotaline-induced pulmonary hypertension in rats. Growth Factors. 12:121-130.

9. Potgens, A.J., Westphal, H.R., de Waal, R.M., and Ruiter, D.J. 1995. The role of vascular permeability factor and basic fibroblast growth factor in tumor angiogenesis. Biol. Chem. Hoppe-Seyler. 376:57-70.

10. Smith, K., et al. 1999. Upregulation of basic fibroblast growth factor in breast carcinoma and its relationship to vascular density, oestrogen receptor, epidermal growth factor receptor and survival. Ann. Oncol. 10:707-713.

11. Benezra, M., Vlodavsky, I., Ishai-Michaeli, R., Neufeld, G., and Bar-Shavit, R. 1993. Thrombininduced release of active basic fibroblast growth factor-heparan sulfate complexes from subendothelial extracellular matrix. Blood. 81:3324-3331.

12. Buczek-Thomas, J.A., and Nugent, M.A. 1999. Elastase-mediated release of heparan sulfate proteoglycans from pulmonary fibroblast cultures. A mechanism for basic fibroblast growth factor (bFGF) release and attenuation of bfgf binding following elastase-induced injury. J. Biol. Chem. 274:25167-25172.

13. George, S.J., Johnson, J.L., Smith, M.A., and Jackson, C.L. 2001. Plasmin-mediated fibroblast growth factor-2 mobilisation supports smooth muscle cell proliferation in human saphenous vein. J. Vasc. Res. 38:492-501.

14. Thompson, K., and Rabinovitch, M. 1996. Exogenous leukocyte and endogenous elastases can mediate mitogenic activity in pulmonary artery smooth muscle cells by release of extracellularmatrix bound basic fibroblast growth factor. J. Cell. Physiol. 166:495-505.

15. Dionne, C.A., Jaye, M., and Schlessinger, J. 1991. Structural diversity and binding of FGF receptors. Ann. N. Y. Acad. Sci. 638:161-166.

16. Inoue, Y., et al. 2002. Basic fibroblast growth factor and its receptors in idiopathic pulmonary fibrosis and lymphangioleiomyomatosis. Am. J. Respir. Crit. Care Med. 166:765-773.

17. Powell, P.P., et al. 1998. Differential expression of fibroblast growth factor receptors 1 to 4 and ligand genes in late fetal and early postnatal rat lung. Am. J. Respir. Cell Mol. Biol. 19:563-572.

18. Sher, I., et al. 2000. Identification of residues important both for primary receptor binding and specificity in fibroblast growth factor-7. J. Biol. Chem. 275:34881-34886.

19. Benisty, J.I., et al. 2004. Elevated basic fibroblast growth factor levels in patients with pulmonary arterial hypertension. Chest. 126:1255-1261.

20. Wedgwood, S., et al. 2007. Fibroblast growth factor2 expression is altered in lambs with increased pulmonary blood flow and pulmonary hypertension. Pediatr. Res. 61:32-36.

21. Molteni, A., Ward, W.F., Ts'ao, C.H., and Hinz, J.M. 1989. Monocrotaline-induced cardiopulmonary injury in rats. Modification by the neutrophil elastase inhibitor SC39026. Biochem. Pharmacol. 38:2411-2419.

22. Molteni, A., Ward, W.F., Ts'ao, C.H., and Solliday, N.H. 1986. Monocrotaline-induced cardiopulmonary damage in rats: amelioration by the angiotensin-converting enzyme inhibitor CL242817. Proc. Soc. Exp. Biol. Med. 182:483-493.

23. Molteni, A., Ward, W.F., Ts'ao, C.H., and Solliday, N.H. 1989. Monocrotaline pneumotoxicity in mice. Virchows Arch. B Cell Pathol. Incl. Mol. Pathol. 57:149-155.

24. Pan, L.C., Wilson, D.W., Lame, M.W., Jones, A.D., and Segall, H.J. 1993. COR pulmonale is caused by monocrotaline and dehydromonocrotaline, but not by glutathione or cysteine conjugates of dihydropyrrolizine. Toxicol. Appl. Pharmacol. 118:87-97.

25. Baird, A., and Ling, N. 1987. Fibroblast growth factors are present in the extracellular matrix pro- duced by endothelial cells in vitro: implications for a role of heparinase-like enzymes in the neovascular response. Biochem. Biophys. Res. Commun. 142:428-435

26. Vlodavsky, I., et al. 1987. Endothelial cell-derived basic fibroblast growth factor: synthesis and deposition into subendothelial extracellular matrix. Proc. Natl. Acad. Sci. U. S. A. 84:2292-2296.

27. Cucina, A., et al. 2002. Autocrine production of basic fibroblast growth factor translated from novel synthesized mRNA mediates thrombininduced mitogenesis in smooth muscle cells. Cell Biochem. Funct. 20:39-46.

28. Lee, T.H., et al. 2006. Heterogeneity of capillary endothelial cells for basic fibroblast growth factor-induced paracrine signaling. Endothelium. 13:191-203.

29. Billottet, C., Elkhatib, N., Thiery, J.P., and Jouanneau, J. 2004. Targets of fibroblast growth factor 1 (FGF-1) and FGF-2 signaling involved in the invasive and tumorigenic behavior of carcinoma cells. Mol. Biol. Cell. 15:4725-4734.

30. Gabrilove, J.L. 2001. Angiogenic growth factors: autocrine and paracrine regulation of survival in hematologic malignancies. Oncologist. 6(Suppl. 5):4-7.

31. Giavazzi, R., et al. 2003. Distinct role of fibroblast growth factor- 2 and vascular endothelial growth factor on tumor growth and angiogenesis. Am. J. Pathol. 162:1913-1926.

32. Nguyen, M., et al. 1994. Elevated levels of an angiogenic peptide, basic fibroblast growth factor, in the urine of patients with a wide spectrum of cancers. J. Natl. Cancer Inst. 86:356-361.

33. Guignabert, C., et al. 2005. Serotonin transporter inhibition prevents and reverses monocrotalineinduced pulmonary hypertension in rats. Circulation. 111:2812-2819.

34. van Suylen, R.J., Smits, J.F., and Daemen, M.J. 1998. Pulmonary artery remodeling differs in hypoxia- and monocrotaline-induced pulmonary hypertension. Am. J. Respir. Crit. Care Med. 157:1423-1428.

35. Pardo, O.E., et al. 2006. FGF-2 protects small cell lung cancer cells from apoptosis through a complex involving PKCepsilon, B-Raf and S6K2. EMBO J. 25:3078-3088.

36. Xiao, D., et al. 2008. Inhibition of fibroblast growth factor 2-induced apoptosis involves survivin expression, protein kinase C alpha activation and subcellular translocation of Smac in human small cell lung cancer cells. Acta Biochim. Biophys. Sin. (Shanghai). 40:297-303.

37. Kennedy, S.H., et al. 1997. Basic FGF regulates 
interstitial collagenase gene expression in human smooth muscle cells. J. Cell. Biochem. 65:32-41.

38. Holnthoner, W., Kerenyi, M., Groger, M., Kratochvill, F., and Petzelbauer, P. 2006. Regulation of matrilysin expression in endothelium by fibroblast growth factor-2. Biochem. Biophys. Res. Commun. 342:725-733.

39. Pintucci, G., et al. 2003. Induction of stromelysin-1 (MMP-3) by fibroblast growth factor-2 (FGF-2) in FGF-2-/- microvascular endothelial cells requires prolonged activation of extracellular signal-regulated kinases-1 and -2 (ERK-1/2). J. Cell. Biochem. 90:1015-1025.

40. Besser, D., Presta, M., and Nagamine, Y. 1995. Elucidation of a signaling pathway induced by FGF-2 leading to uPA gene expression in NIH $3 \mathrm{~T} 3$ fibroblasts.
Cell Growth Differ. 6:1009-1017.

41. Ribatti, D., et al. 1999. In vivo angiogenic activity of urokinase: role of endogenous fibroblast growth factor-2. J. Cell Sci. 112:4213-4221.

42. Naik, M.U., Vuppalanchi, D., and Naik, U.P. 2003 Essential role of junctional adhesion molecule- 1 in basic fibroblast growth factor-induced endothelial cell migration. Arterioscler. Thromb. Vasc. Biol. 23:2165-2171.

43. Merklinger, S.L., Jones, P.L., Martinez, E.C., and Rabinovitch, M. 2005. Epidermal growth factor receptor blockade mediates smooth muscle cell apoptosis and improves survival in rats with pulmonary hypertension. Circulation. 112:423-431.

44. Schermuly, R.T., et al. 2005. Reversal of experimental pulmonary hypertension by PDGF inhibition.
J. Clin. Invest. 115:2811-2821.

45. Eddahibi, S., et al. 2001. Serotonin transporter overexpression is responsible for pulmonary artery smooth muscle hyperplasia in primary pulmonary hypertension. J. Clin. Invest. 108:1141-1150.

46. Schalling, M., Dagerlind, A., Stieg, P., Lindquist, C., and Hokfelt, T. 1991. Colocalization of neurotransmitters analyzed by in situ hybridization. Eur. Neuropsychopharmacol. 1:173-176.

47. Raj, T., et al. 2006. Inhibition of fibroblast growth factor receptor signaling attenuates atherosclerosis in apolipoprotein E-deficient mice. Arterioscler. Thromb. Vasc. Biol. 26:1845-1851.

48. Carson, F.L. 1997. Histotechnology: a self-instructional text. American Society for Clinical Pathologists Press. Chicago, Illinois, USA. 304 pp. 